\title{
Exploiting Contexts with House Log for Value-Added Smart TV Services
}

\author{
Tetsuya Masuda, Yuichi Watanabe, Shinsuke Matsumoto, Sachio Saiki, Masahide Nakamura \\ Graduate School of System Informatics, Kobe University \\ 1-1 Rokkodai Nada, Kobe, Hyogo 657-8501, Japan \\ Email: masuda@ws.cs.kobe-u.ac.jp
}

\begin{abstract}
We have been studying log contexts using largescale house log collected in home network system (HNS). A log context is defined with both the current and past situations of a house, where the current situation is characterized by the current status of an appliance (or a sensor), while the past situations are derived from the house log. In this paper, we propose contextaware services for smart TVs using the log contexts within HNS. We first present three different types of log contexts derived from smart TV. We then discuss value-added services using such log contexts, assuming that a smart TV is integrated with HNS.
\end{abstract}

\section{INTRODUCTION}

Research and development of smart TVs are recently attracting great attention in the field of consumer electronics. Major TV manufacturers are developing various technologies and products, and put them on the market. A smart TV can be considered as an information appliance (or a computer system) that allows a user to install and run advanced applications. Hence, it is often regarded as a self-contained platform powerful enough to provide audio/visual and information services.

However, considering that a smart TV is installed in a house, it can be integrated with other household appliances and information in a house, which allows more valuable and smarter services. The integration is feasible with the emerging home network system (HNS) (also called smart home) [1].

We have been extensively studying context-aware services in HNS, which autonomously execute (or recommend) appropriate actions based on situational information in a house. The situational information is called context, and is captured by sensors of HNS. To enable more advanced contexts, we proposed to use house log in [2], which is history of data acquired from sensors and appliances of HNS. Using the house $\log$, we can define a new type of context, called log context. A log context is defined with both the current and past situations.

In this paper, we propose context-aware services for/with smart TVs using such log contexts defined in HNS. To propose new services, we first enumerate log contexts that can be derived from smart TVs. For this, we consider three types of log contexts: (1) contexts derived by a smart TV only, (2) contexts derived from a smart TV and other appliances, (3) contexts derived from multiple smart TVs in a community. We then discuss the context-aware services with the log contexts, assuming that a smart TV is integrated with HNS.

\section{Context-Aware Services Using House Log [2]}

A context-aware service is a service that autonomously executes appropriate actions when a context is established. A context is usually defined by situational information characterized by sensors. For example, using a temperature sensor, we can define a context "it is cold" by a condition that "the value of the temperature sensor is less than 8 degree". A contextaware service automatically turns on a heater when it is cold. Traditional context-aware services consumed only interesting data to evaluate the context. All irrelevant data were discarded without being stored, as the storage was limited and expensive.

Now in the era of big data, we can manage large-scale data with inexpensive storage. It is realistic to store any kinds of data from HNS as house log. This motivated us to use such the house $\log$, in order to extend the conventional contexts so as to consider past situations. The new type of context is called $\log$ context [2]. For example, log data of a temperature sensor enables log contexts like: "it is colder than yesterday", or "it is the coldest Xmas for 3 years". Using the house log, we can implement smarter services. For example, a context-aware service automatically raises the temperature setting of a heater, when it is colder than yesterday.

Our interest in this paper is to investigate log contexts and context-aware services, relevant to smart TVs. Specifically, we consider log contexts that can be defined by log data of a smart $\mathrm{TV}$. We also consider the context-aware services using the log contexts of the smart TV.

\section{LOG CONTEXTS DERIVED FROM SMART TV}

\section{A. Log Context Derived from Single Smart TV}

Log data of a smart TV includes time-stamped records of operations, watched programs and device status. Using such log data, log contexts that characterize user's behaviors and preferences can be defined. For example:

Log Contexts for Viewing Time: By searching the log data where a smart TV is turned on, it is possible to identify the date and hour when a user was watching the smart TV. We can also calculate the viewing time. A log context "Yesterday, I was watching a TV more than half a day" can be defined by a situation that the total viewing time in yesterday was more than 12 hours. Similarly, using the log of the last week, we can define a log context "I'm watching a TV at the same period of time as the last week".

Log Contexts for Watched Programs: Using a history of watched channels and programs, we can define log contexts characterizing user's preference. A log context "You are now watching the mostly-viewed channel in the last week" can be evaluated by statistics of the channel $\log$ in the last week. A 
$\log$ context "You are watching this program every week for this month" can be derived from the program log in this month.

Log Contexts for TV Setting: The log data of a smart TV may involve a history of TV operations, which reflects user's preference to TV setting. For example, by investigating log of sound volume, a log context "I'm always watching movies with loud sound" can be evaluated. Similarly, we can define a $\log$ context "I usually watch news with English subtitles" using the operation $\log$ of subtitles.

\section{B. Log Contexts Defined with Other Appliances}

Integrating the log data of a smart TV with $\log$ data of other appliances, we can define more advanced log contexts.

Log Contexts Detecting Wasted Energy: Integrating log of a smart TV and $\log$ of an appliance that is mutually exclusively used, we can derive log contexts that detect energywasting behaviors. For example, integrating log of a game console, a log context "A TV program is watched while a game console is left on for a long time" can be defined.

Log Contexts Reflecting Motivation: Integrating log of household appliances with the log of a smart TV may characterize user's actions motivated by a TV program. For example, integrating log of a vacuum cleaner, we can define a $\log$ context "A vacuum cleaner is turned on soon after a TV program of house cleaning was viewed" can be defined, which characterizes how the TV program affects user's daily life.

\section{Log Contexts with Global Information}

The emerging technologies for smart city [3] will enable a smart TV to consume global information gathered from other houses or infrastructures of a city. Such information allows us to define $\log$ contexts reflecting the status of the community.

Log Contexts for Trends in Community: Investigating log data of multiple smart TVs in a town, a log context "This program is most popular in this town" can be defined. Also, analyzing the log data for each time period, a context like "In this town, the news program is mostly viewed in between 7 and $8 \mathrm{pm}$ " can be defined. These contexts reflect community's trends and preferences for the TV program.

Log Contexts for Viewing Environment: Gathering environmental data (e.g., sound volume, brightness, temperature, etc.) from various houses, we may estimate the best environment setting for viewing a movie on a smart TV. A log context with such estimated setting may promote presence of contents and user's satisfaction.

\section{USING LOG CONTEXTS FOR SMART TV SERVICES}

We here propose several service scenarios of smart TV services using the log contexts.

Stuck-On-TV Warning Service: Using the log context "Yesterday, I was watching a TV more than half a day", this service displays a warning message "Do not watch a TV too long today" on the smart TV. The service may automatically turn off the TV to consider user's health.

Wasting Energy Detection Service: Using the log contexts of a smart TV and related appliances, this service detects and alerts wasting energy. For example, when the log context "A TV program is watched while a game console is left on for a long time" is satisfied, the service prompts a message on a smart TV to turn off the game console. More detailed information like the time period of waste or the amount of wasted energy would encourage the user to perform the energysaving behaviors.

Housework Encouraging Service: Using the log contexts of household appliances and a smart TV, this service encourages the user to do housework. For example, when the service detects the long absence of house cleaning from the log of a vacuum cleaner, the service plays back a house cleaning TV show on a smart TV to encourage the user. When the $\log$ context "A vacuum cleaner is turned on soon after a TV program of house cleaning was viewed" is satisfied, the service praises the user with a message or contents. Thus, the user realizes the importance of the housework.

Communication Promotion Service: Using the log context for trends in community, this service promotes a change of communication. Specifically, using the log context "this program is most popular in the town", the service recommends users to watch the program, with showing the statistics of viewers in the community. Watching a popular TV program produces common topics, which may promote better opportunities to communicate with others.

Best Viewing Environment Service: Using the log contexts for viewing environment, this service estimates the best viewing environment setting. Depending on the TV program to be viewed, the service operates the smart TV for optimal sound volume and brightness. The service may use other appliances such as speakers, lights and an air-conditioner, to produce best settings of surrounding.

\section{CONCLUSION}

In this paper, we have discussed log contexts that can be derived from a smart TV. We have also proposed value-added services for a smart TV using the log contexts. It was shown that sophisticated contexts were defined by integrating log data from other appliances or global information. Applications of such $\log$ contexts are not limited within the entertainment of a smart TV. They are promising for improving user's quality of life, as well as for constructing better community.

Acknowledgment: This research was partially supported by the Japan Ministry of Education, Science, Sports, and Culture [Grant-in-Aid for Scientific Research (C) (No.24500079, No.24500258), Scientific Research (B) (No.26280115), Young Scientists (B) (No.26730155)] and Kawanishi Memorial ShinMaywa Education Foundation.

\section{REFERENCES}

[1] M. Nakamura, et al., "Constructing home network systems and integrated services using legacy home appliances and web services," Journal of Web Services Research, vol. 5, no. 1, pp. 82-98, 2008.

[2] Y. Watanabe, et al., "Studying Context-Aware Services Using House Log in Home Network System," Technical Report of IEICE, vol. 113, no. 326, LOIS2013-37, pp. 77-82, Nov. 2013 (in Japanese).

[3] S. Yamamoto, et al., "Using Cloud Technologies for Large-Scale House Data in Smart City," Int'l. Conf. on Cloud Computing Technology and Science (CloudCom2012), pp.141-148, Dec. 2012. 